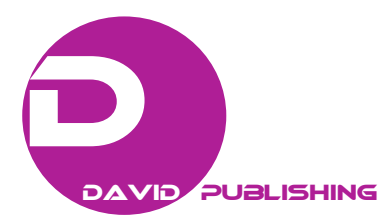

\title{
What Does TLC Mean?
}

\author{
Krystyna Tyrpień-Golder \\ Department of Chemistry, School of Medicine with the Division of Dentistry in Zabrze, Medical University of Silesia in Katowice, \\ Jordana 19, 41-808 Zabrze, Poland
}

\begin{abstract}
Among various meanings of TLC like The Last Cigarette - a smoking cessation program that offers numerous resources and tools to assist smokers in their quit efforts, there is also Thin Layer Chromatography, an analytical technique, which can be useful to check the process of quitting smoking and allow to observe differences in nicotine metabolism. The task of this research was checking sufficiency of thin layer chromatography followed by densitometry for monitoring process of cutting down on smoking. Assembled data suggested that during one week after stopping smoking small amounts of main nicotine metabolites were still being removed with urine from the organism. The chromatographic analysis of nicotine and its main metabolites are multi-stage process which is characterized by about $80 \%$ recoveries but a satisfactory reproducibility (2.8\%-9.2\%). These results also depend on stationary phases applied for TLC/HPTLC (High Performance Thin Layer Chromatography) and problems connected with material applied to separate main nicotine metabolites have been solved. If the chromatographic plates for TLC used for this research were properly marked by HPLTC, confusion caused by various meaning of TLC abbreviation would be eliminated.
\end{abstract}

Key words: Thin layer chromatography, chromatographic plates, separation, tobacco smoke, densitometry.

\section{Introduction}

TLC can be joined with Association like Toyota Land Cruiser Owners Club aims to promote the culture of automotive, motorsport, automotive tourism and road safety rules as well as charity events e.g. annual Finale of the Great Orchestra of Christmas Help in Poland ${ }^{1}$.

TLC is also connected with many IT companies operating in industry, servicing constructions and giving engineering solution $2,3,4$, moreover comprehensive customer service connected with bearings in Poland (TLC Łożyska Sp.J.) $)^{5}$. Of all the meanings of TLC can be distinguished, those related to education goal like language school-Trinity Lutheran College, located in Everett, WA ${ }^{6}$. It is a Christian

Corresponding author: Krystyna Tyrpień-Golder, Ph.D., associate professor, research fields: toxicology and chromatography.

${ }^{1}$ Society Toyota Loyalty Club (TLC), Owners Club, Poland, www.tlc.org.pl

2 TLC Marketing Worldwide, http, www.tlcmarketing.com/uk/

3 Telecom Italia, www.telecomitalia.com

4 TLC Electrical Supplies , www.tlc-direct.co.uk

${ }^{5}$ Centre Bearing, www.tlc.pl/pr.swf (in polish)

6 Trinity Lutheran College, http://www.tlc.edu liberal arts college offering degrees in Business, Communications, Graphic Design and Psychology. In 1980, TLC television channel was launched and this television network recommends exclusive photos, videos and full episodes, games and more finding fun and beauty in many countries.

For children, TLC means The Leaky Cauldron, it is a Harry Potter fan site and $\mathrm{blog}^{7}$. On line TLC is also joined with The Library Corporation ${ }^{8}$.

On the other hand, TLC is the worldwide organization focused on treatment, research, and outreach for people with trichotillomania, skin picking and related disorders. It is connected with Therapeutic Lifestyle Changes (TLC) ${ }^{9}$.

Among them, the guidelines specific to Health Link members who are a part of BJC Health Care, is also connected with TLC, and it means The Last

\footnotetext{
7 The Leaky Cauldron, www.the-leaky-cauldron.org/ The Learning Company Workbooks, www.hmhco.com/learning-company/tlc-workbook 9 Therapeutic Lifestyle Changes (TLC) for High Cholesterol Topic Overview, http://www.webmd.com/cholesterol-management/tc/therapeutic -lifestyle-changes-tlc-diet- for-high-cholesterol
} 
Cigarette $^{10}$.

For analysts, it is obvious that TLC (Thin Layer Chromatography) is separation process of mixture which can be performed in a plane (planar chromatography) with densitometric detection [1].

These two last abbreviations can be joined with cigarette smoking, and especially with smoking cessation processes. In our research, planar chromatography was applied for assessment of tobacco smoke exposure to different group of people from Silesia Region in Poland by the use of earlier described chromatographic conditions [2,3]. Nicotine and its metabolites are rather really determined by planar chromatography [4-6], most often immunoassay has been used for screening and HPTLC for detail analysis [7].

The aim of this paper was to underline various meaning of TLC which has been used to evaluate tobacco smoke exposure and problems during monitoring of cessation process in reverse phase of chromatographic system.

\section{Materials and Methods}

S-(-)-nicotine (1-methyl-2-[3-pyridyl] pyrrolidine), S-(-)-cotinine (1-methyl-5-[3-pyridyl]-2-pyrrolidinone) from Sigma-Aldrich and 3 'R, 5'S-trans-3'-hydroxycotinine of Toronto Research Chemicals Inc.. (Ontario, Canada). Non-polar resin Amberlite XAD-2 was obtained from Supelco.

All chemicals were of analytical grade. Dichloromethane (Baker, Germany), methanol, acetone and acetonitrile (POCh, Gliwice, Poland) with a purity of spectroscopy or HPLC.

At the beginning of our research, we separated both main nicotine metabolites: cotinine and trans-3'-hydroxycotinine on TLC plates no 818145 Alugram aluminium RP-18 W/UV254, $0.15 \mathrm{~mm}, 5 \times$ $20 \mathrm{~cm}$, Macherey Nagel, Düren, Germany.

The separations were performed in a horizontal sandwich chamber (DSII, Chromdes, Lublin, Poland).

${ }^{10}$ Health Link, https://www.healthlink.com/health_tlc.asp
Visualization of the chromatograms was carried out at a wavelength $\lambda=254 \mathrm{~nm}$ under UV lamp, next by a densitometer (CS 9301 Shimadzu, Japan) at $\lambda=260$ $\mathrm{nm}$ and "zigzag" scanning mode, according to described earlier procedure [2]. This chromatographic system has been applied to analyze nicotine metabolites in urine of children, in serum of pregnant women and their new born babies, smokers as well as people exposed to second hand smoke and in the case of cessation process monitoring [7].

The chromatographic system (Fig. 1) consisted of RP-18 stationary phase and acetonitrile and water $(88+$ $12, \mathrm{v} / \mathrm{v}+50 \mathrm{mg} / \mathrm{mL}$ sodium octanosulfonate) as mobile phase and $8 \mathrm{~cm}$ distance as well as detection at UV absorbance $(\lambda=260 \mathrm{~nm})$ was sufficient to separate not only cotinine from trans-3'-hydroxycotinine, but also allowed to determine in some cases nicotine and its oxide Fig. 2.

Moreover, this analytical technique (TLC with densitometry) has also been applied in similar studies carried out to assess exposure to tobacco smoke among pregnancies, children, and students of medicine from Upper Silesia Region in Poland [7-9]. Application of this chromatographic system allowed to control cessation process of volunteer and determine which of nicotine metabolite will be eliminated with urine for long period of time (Fig. 3) [6].

TLC can have various meanings for different groups of societies and for people in different countries. But it can also be problematic for chromatographers. After delivery of the next new batch of chromatography plates to carry out the separation of the fraction containing nicotine and its metabolites at the same chromatographic system, we did not receive the expected results (Fig. 1). The longer distance to develop the chromatogram (Fig. 1) did not improve separation results. If these plates were labeled differently than previously used by us, the modification of the chromatographic system to allow the separation of the major nicotine metabolites would have been easier and we would have save the time and required for that purpose reagents. 


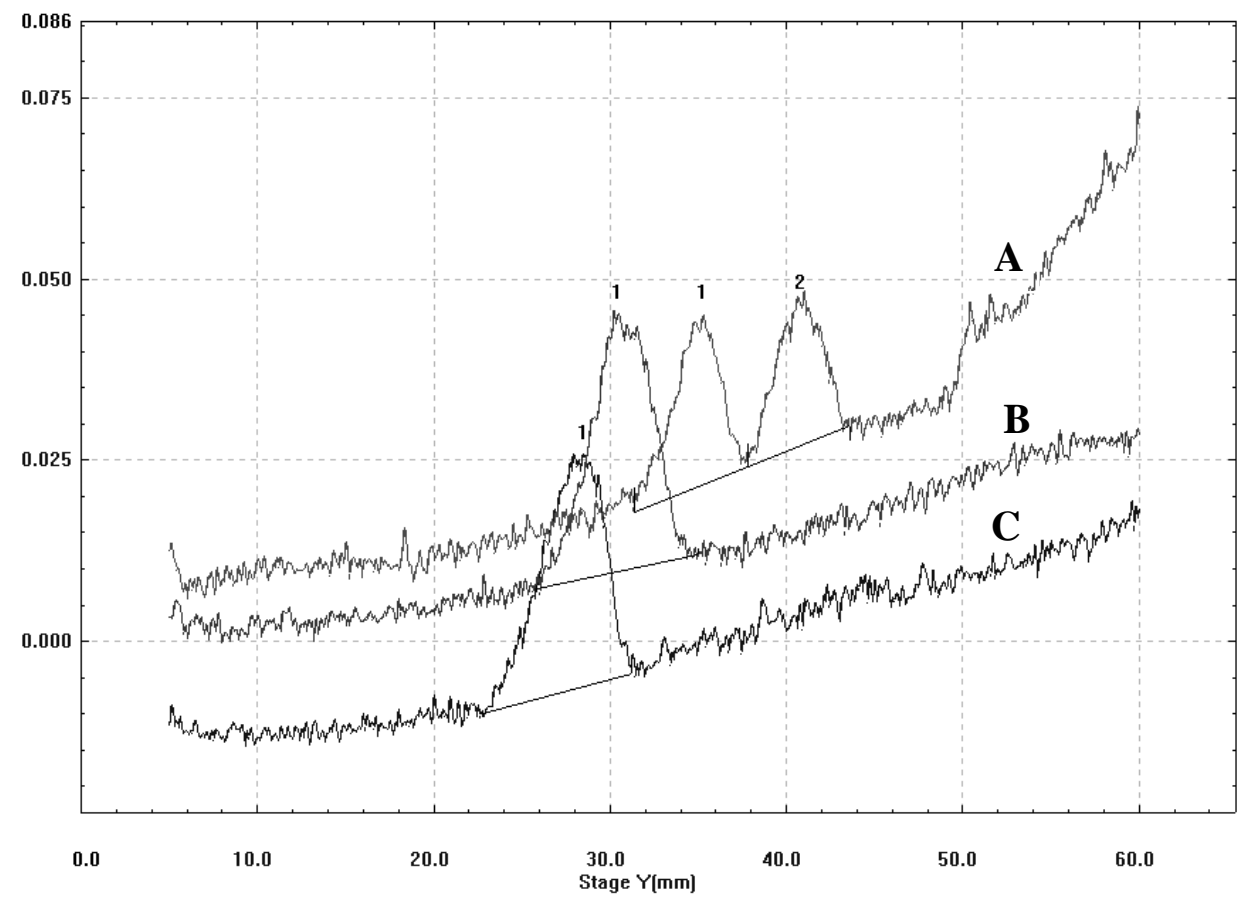

Fig. 1 HPTLC RP18 plate/acetonitrile-water $(88+12, \mathrm{v} / \mathrm{v}+50 \mathrm{mg} / \mathrm{mL}$ sodium octanosulfonate $)$ UV absorbance at $\lambda=260 \mathrm{~nm}$. (A) developing distance of $8 \mathrm{~cm}$ : 1-cotinine and 2-trans-hydroxycotinine; (B) developing distance of $10 \mathrm{~cm}$ (standards do not separate); (C) developing distance of $8 \mathrm{~cm}$ (standards do not separate).

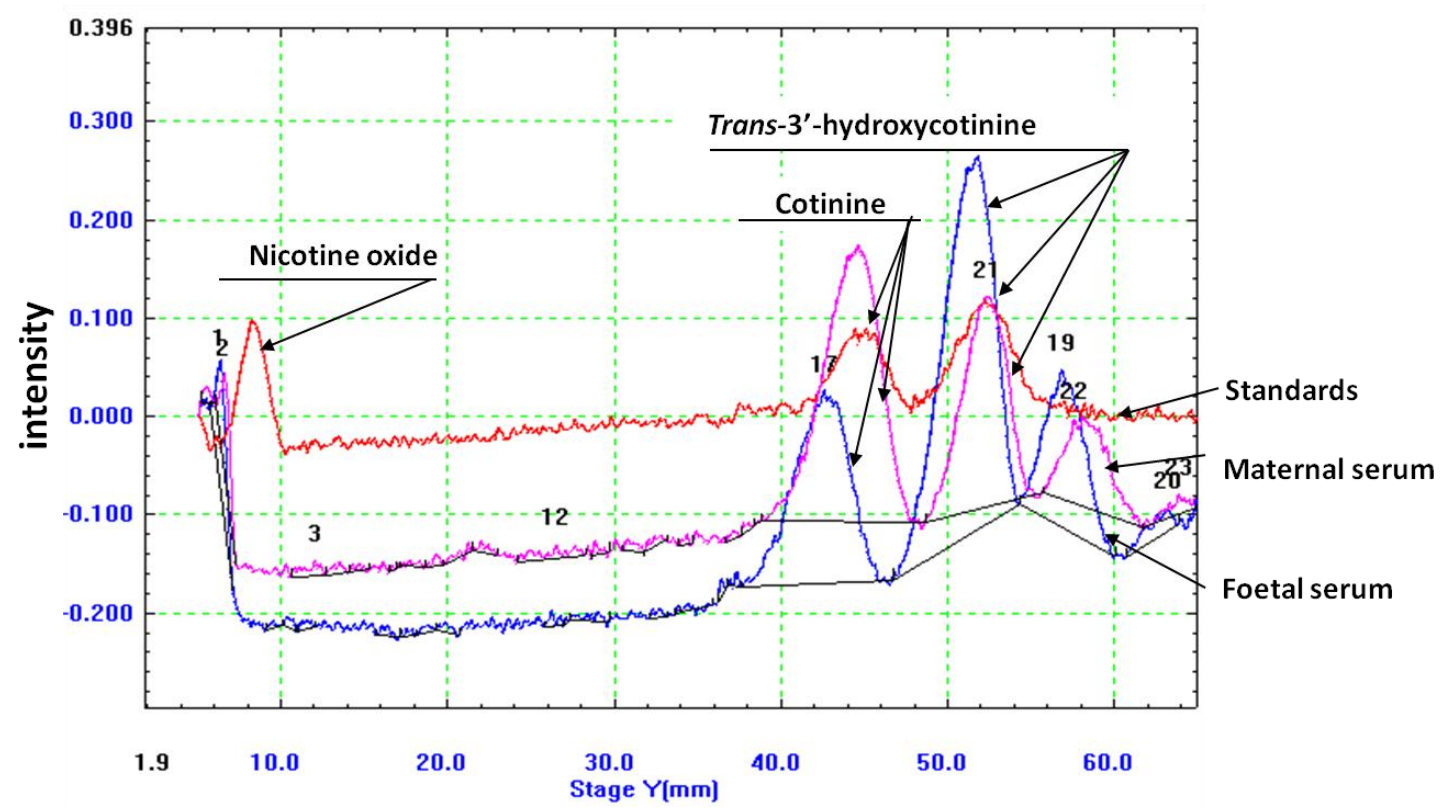

Fig. 2 Comparison of the main nicotine metabolites concentration isolated from maternal and fetal serum determined by TLC with densitometry [7].

It was shown that only a modification of the mobile phase like acetonitrile-water $(80+20, v / v)$ allowed to obtain similar results to those obtained previously (Fig. 4).
A number of studies [2-4, 6] with the designation for nicotine metabolites in biological material (urine, serum, amniotic fluid) have used reverse phase TLC/HPTLC plates coated with nano silica with partial 


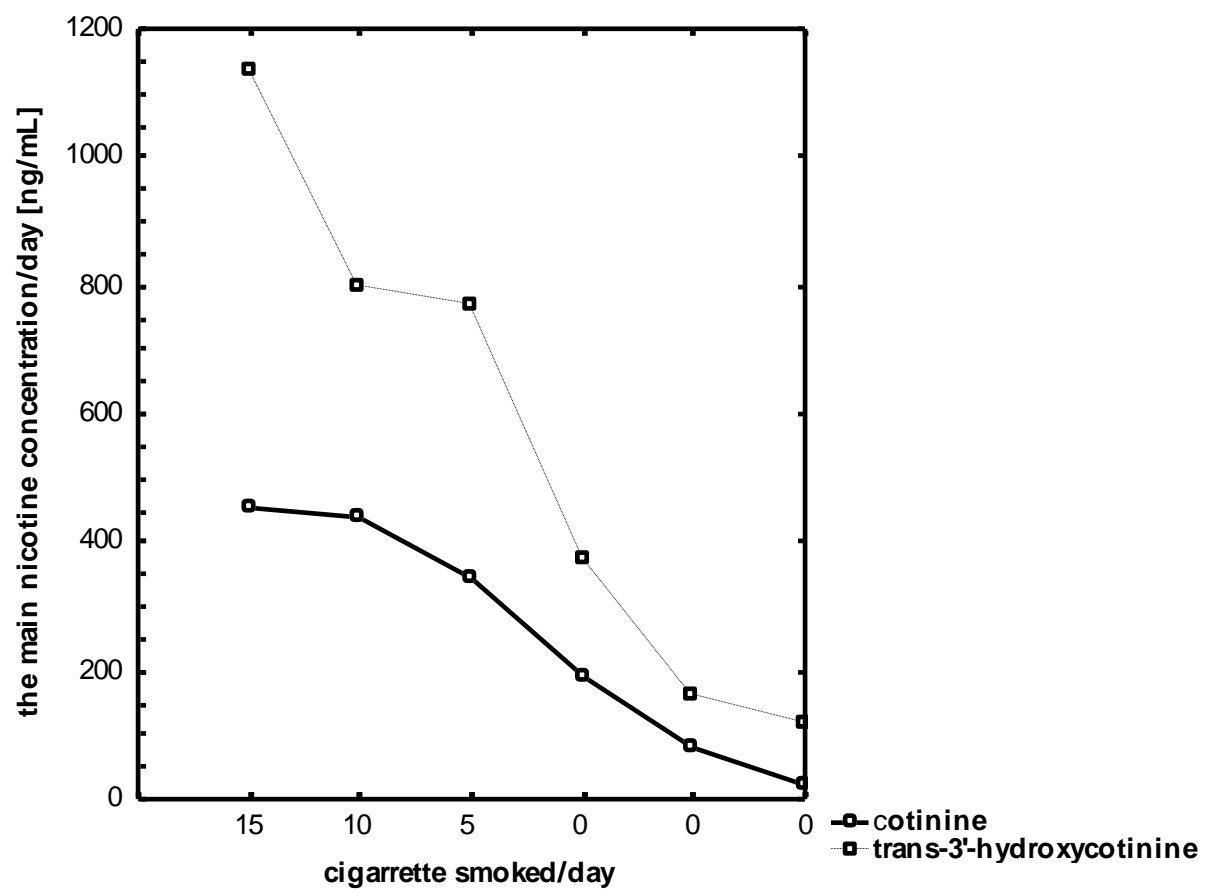

Fig. 3 Dependence between main nicotine metabolites concentration and amount of cigarette/day in smoking cessation process determined by HPTLC with densitometry [7].

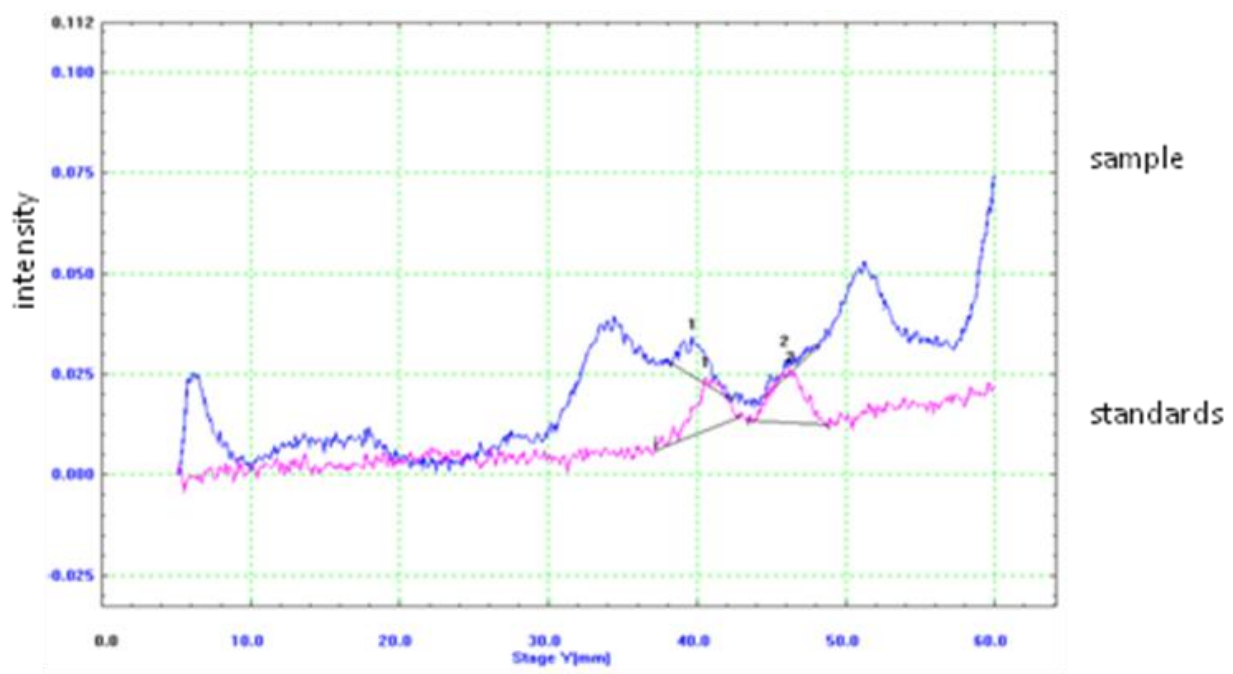

Fig. 4 Densitogram of the urinary extract of children with ASD (Autism Spectrum Disorder) in which main nicotine metabolites were identified: 1-cotinine, 2-trans-hydroxycotinine; separated on stationary phase C18 HPTLC plates $5 \times 10 \mathrm{~cm}$ (Macherey-Nagel). As the mobile phase a mixture of acetonitrile and water $(80: 20, \mathrm{v} / \mathrm{v})$ supplemented with sodium octasulfonate.

octadecyl modification C18 $-0.15 \mathrm{~mm}$, wettable with water with a mobile phase acetonitrile-water $(88+12$, $\mathrm{v} / \mathrm{v})$ and have found individual differences in metabolizing nicotine (Fig. 2), as well as differences in their excretion. It can be also useful in monitoring of cigarette smoking cessation as well as in metabolomic research.

Reproducibility of these assays depends on type of used chromatographic plates. However, the analysis of nicotine and its main metabolites are a multi-stage procedure, which is characterized by not so high recoveries (about 80\%) but a satisfactory 
reproducibility (2.8 the last plates applied up to $9.2 \%$ earlier used). Any changes in the procedure for the preparation of the stationary phase should result in changes in the designation of a new product. Using the same part number and labels promotes misunderstanding between technicians and those who interprets the results.

\section{Conclusions}

If the chromatographic plates using for this research were properly marked by HPLTC (High Performance Thin Layer Chromatography), confusion caused by various meaning of TLC abbreviation would be eliminated as well. In addition, catalog numbers should be differentiated in the case of every technological changes of chromatographic products.

\section{Acknowledgement}

This paper is dedicated to M. Sc. Eng. Cezary Dobosz for his technical support and M.Sc. Grażyna Garłowska for the supply of urine samples during cigarette cessation.

\section{References}

[1] Tyrpień-Golder, K. 2013. "Application of Scanning Densitometer for Environmental and Biomedical Research.” ABiD 18 (4): 269-75.

[2] Tyrpień, K. 2006. "Analysis of Chosen Organic Tobacco Smoke Components and Their Metabolites by Planar Chromatography." Polish Journal of Environmental
Studies 15 (4): 609-14.

[3] Tyrpień, K., Bodzek, D., Janoszka, B., Dobosz, C., and Stęplewski, Z. 2000. "Application of Liquid Separation Techniques to the Determination of the Main Urinary Metabolites." Journal of Chromatography A 870 (1-2): 29-38.

[4] Bazylak, G., Brózik, H., and Sabanty, W. 2000. "HPTLC Screening Assay for Urinary Cotinine As Biomarker of Environmental Tobacco Smoke Exposure among Male Adolescents." Journal of Pharmaceutical and Biomedical Analysis (24): 113-23.

[5] Musharraf, G., Shoaib, M., and Najam-ul-Haq, S. 2011. "TLC-Densitometric Method Development and Validation for the Quantification of Nicotine in Tobacco Smoked, Sniffing, Dipping, and Chewing Products." JPC-Journal of Planar Chromatography-Modern TLC 24 (5): 381-7.

[6] Paillat, L., Perichet, C., Lavoine, S., Meierhenrich, U., and Fernandez, X. 2012. "Performance Thin-Layer Chromatographic Method for the Determination of Nicotine in Tobacco (Nicotiana tabaccum L.) Extracts." Journal of Planar Chromatogr.-Modern TLC 25 (6): 23-9.

[7] Tyrpień, K. 2008. "The Use of Planar Chromatography to Assess Exposure to Selected Components of Tobacco Smoke." Ph.D. thesis, Medical University of Silesia, Katowice, Poland. (in Polish)

[8] Tyrpień, K., Bodzek, P., and Mańka, G. 2001. "Application of Planar Chromatography to the Determination of Cotinine in Urine of Active and Passive Smoking Pregnant Women." Biomedical Chromatography 5 (1): 50-5.

[9] Szumska, M., Tyrpień, K., Kowalska, M., Wielkoszyński, T., and Dobosz, C. 2013. "Medicine Students and Exposure to Environmental Tobacco Smoke". International Journal Occupational Medicine Environmental Health 26 (2): 313-20. 